\title{
The Effect of Particle Concentration of Furnace Black on Field Intensity in the Air Gap of an Electric Precipitator
}

\author{
I. Kh. Khairullin ${ }^{a, *}$, M. V. Okhotnikov ${ }^{a}$, A. A. Nusenkis ${ }^{b}$, T. A. Volkova ${ }^{a}$, \\ D. V. Maksudov ${ }^{a}$, and D. U. Saigafarov ${ }^{a}$ \\ ${ }^{a}$ Ufa State Aviation Technical University, Ufa, Bashkortostan, 450008 Russia \\ ${ }^{b}$ GIP Electro, Ufa, Bashkortostan, 450097 Russia \\ *e-mail:journal-elektrotechnika@mail.ru \\ Received December 19, 2017
}

\begin{abstract}
The results of a study of the development of small-sized electric precipitators obtained by the Department of Electrical Engineering of Ufa State Aviation Technical University are presented.
\end{abstract}

DOI: $10.3103 / \mathrm{S} 106837121801008 \mathrm{X}$

Environmental concerns make the development necessary of compact and efficient electric precipitators (EPs). The air-gap gauge between electrodes is one of the important design parameters that determine the filter performance, as well as the pattern of the electrical field and its density.

At the design stage of an EP, furnace-black particles are not taken into account when analyzing the pattern of an electrical field. The electric conductivity of these particles results in a decrease of breakdown voltage, an increase in currents, and changes in the field pattern of an EP. In addition to experimental studies of the effect of furnace-black particles on field density, it seems worthwhile to carry out a detailed investigation using the Ansys software system. Modeling was performed with a computational model simulating a proportional electrostatic field between plane electrodes with various potentials. Particles with a diameter of 20-40 $\mathrm{nm}$ are comparable with those that appear during combustion of diesel fuel. The concentration of particles depends on type of fuel, combustion process, and system regime. When considering variants with local and mass content in the field studied, it can be observed that a group of particles that have no charge but have conductibility acquire the properties of electric conductor.

As a result of studies, it has been determined that some lines of flux are closed over the surface of particles and the field density along the electrode is locally changed according to the location of the group of particles. An increase in the number of similar groups results in multiple alterations of field-density static values, the appearance of avalanche-type streamers, and electrical breakdown.
A model with a smooth disk serving as a positive electrode and a complete sphere as a negative one was used to determine the breakdown-voltage stage in the presence of furnace-black particles in the flue gas. The model allows one to generate a symmetric field discarding "outside" zones of corona discharge, which is confirmed by the charge-distribution density over the surface of the disk and the maximum level of the field density generated in the disk center, where the distance between electrodes is minimal $\left(\delta_{\text {min }}\right)$. According to data of modeling with $\delta_{\min }=6 \mathrm{~mm}$, the maximum level of field density $E_{\max }=3 \mathrm{kV} / \mathrm{mm}$ is generated with an electrode voltage of $18 \mathrm{kV}$. An electroconductive particle with a diameter of $9 \mu \mathrm{m}$ placed between the electrodes of the studied model decreases breakdown voltage $U_{\mathrm{b}}$ to $15.5 \mathrm{kV}$. When there is no particle and a similar breakdown voltage, $E_{\max }$ comes to little more than $0.26 \mathrm{kV} / \mathrm{mm}$.

The results of experimental verification of the computational model with various concentrations of particles (determined by the smoke number) are presented in this article as well.

If we know the concentration and the size of furnace-black particles in the flue gas, one can control the electrode voltage values of an EP, thus providing its operational efficiency and preventing the occurrence of spark breakdown. The possible breakdown voltage should be determined on a case-by-case basis taking into account all the operational characteristics, parameters, and characteristics of an EP. The obtained results allows one to be more specific concerning the parameters of an EP depending on operating conditions. They can be used in EP design.

Translated by V. Melnikov 